\title{
Therapie von Knochenmetastasen beim Mammakarzinom - Zoledronat senkt Skelettkomplikationen osteolytischer Läsionen wirksamer als Pamidronat
}

Das Mammakarzinom ist eine Erkrankung mit hoher Inzidenz. Allein in Deutschland werden etwa 50000 Brustkrebserkrankungen pro Jahr diagnostiziert. Fast die Hälfte der betroffenen Frauen erleidet eine Krankheitsprogression. Im fortgeschrittenen $\mathrm{Tu}$ morstadium weisen $65-75 \%$ der Patientinnen Knochenmetastasen auf, die zu Skelettkomplikationen (skeletal-related events; SREs) führen können. Diese haben eine große klinische Bedeutung, da sie die Lebensqualität der Patientinnen entscheidend beeinflussen. Das Übergreifen der Erkrankung auf das Skelettsystem kann zu zusätzlichen Schmerzen, längerer Hospitalisierung, Querschnittssymptomatik oder gar, infolge der damit verbundenen Komplikationen, zum Tod des Patienten führen. Die Standardtherapie bei ossären Metastasen des Mammakarzinoms oder Multiplen Myeloms war bisher die intravenöse Gabe des Bisphosphonats Pamidronat. Im Oktober wurden auf dem Jahreskongress der European Society for Medical Oncology (ESMO) in Nizza die Ergebnisse einer Subgruppenanalyse einer Phase-III-Multicenter-Studie zum Multiplen Myelom und Mammakarzinom vorgestellt. Zoledronat (Zometa ${ }^{\circledR}$, Novartis Pharma $\mathrm{GmbH}$, Nürnberg, Deutschland) bewies bei
Patientinnen mit Mammakarzinom und mindestens einer osteolytischen Läsion eine signifikant höhere Wirksamkeit als Pamidronat. In die internationale randomisierte PhaseIII-Studie waren insgesamt 1648 Patientinnen mit osteolytischen und/ oder osteoblastischen Knochenmetastasen aufgrund eines Multiplen Myeloms oder eines Mammakarzinoms eingeschlossen worden. Dabei zeigte sich, dass eine palliative Strahlentherapie bei Patienten unter Zoledronat signifikant seltener notwendig war als bei denen unter Pamidronat $(p=0,031)$. Die Verträglichkeit beider Substanzen war vergleichbar.

Auf dem ESMO wurden nun Daten zum Mammakarzinom vorgestellt, die neben dem Auftreten der ersten SRE auch berücksichtigen, inwiefern weitere Ereignisse verhindert oder verzögert werden können. Die $766 \mathrm{~Pa}$ tientinnen mit fortgeschrittenem Mammakarzinom und mindestens einer Knochenmetastase wurden nach Läsionstyp gesondert betrachtet. Die Patientinnen beider Gruppen hatten röntgenologisch gesicherte osteolytische $(n=352)$, osteoblastische $(n=154)$ oder gemischtförmige Knochenläsionen $(n=260)$. Als Therapie erhielten die Frauen über 12 Monate alle 3-4 Wochen entweder $4 \mathrm{mg}$ Zoledronat (378 Patienten) als 15-minütige In-

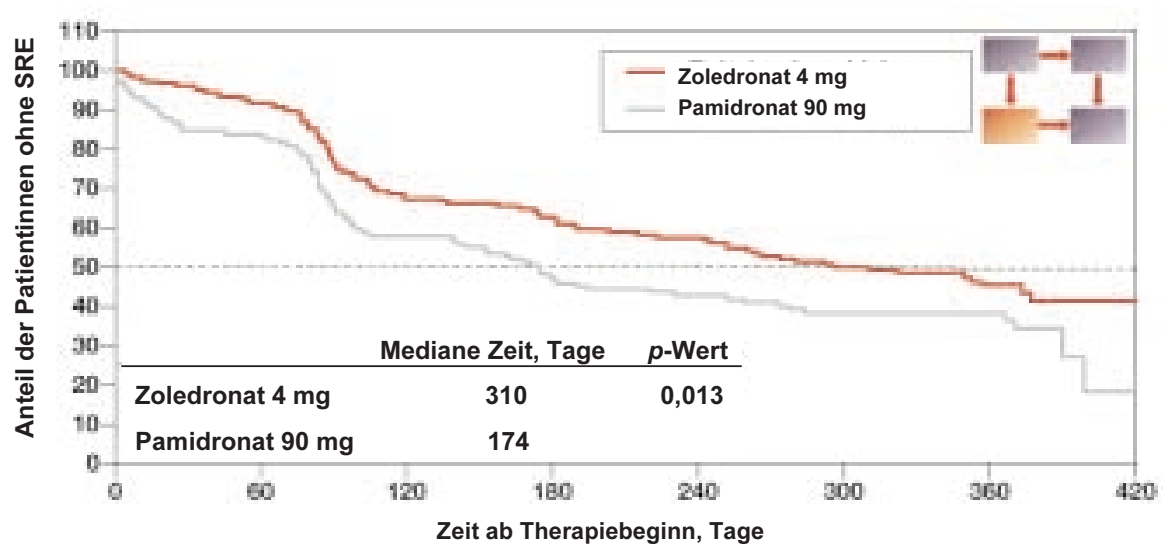

Abb. 1. Kaplan-Meier-Kurve der Zeit bis zum Auftreten der ersten SRE bei Mammakarzinom-Patientinnen mit mindestens einer osteolytischen Läsion. fusion oder $90 \mathrm{mg}$ Pamidronat (388 Patienten) als 2-Stunden-Infusion.

Der primäre Studienendpunkt war der Anteil der Patienten mit mindestens einer SRE nach 13 Monaten. Eine SRE wurde definiert als pathologische Fraktur, Rückenmarkskompression sowie als Notwendigkeit einer Bestrahlung oder Operation des Knochens. Sekundäre Endpunkte waren die Zeit bis zum Auftreten der ersten SRE, die Skelettale Morbiditätsrate (SMR, gemessen als Anzahl der SREs pro Patient und Jahr) und eine «Multiple Event»-Analyse des Risikos einer SRE. In diese Berechnung flossen mehrere Endpunktbetrachtungen der SREs ein: Die Anzahl der SREs, die Zeit bis zur ersten SRE, die Zeit bis zu jeder weiteren SRE und die Zeit zwischen den einzelnen Ereignissen.

\section{Weniger und später auftretende Skelettkomplikationen unter Zole- dronat bei osteolytischen Läsionen}

Auch die osteoblastische oder gemischtförmige Form der Knochenmetastasierung führt zu SREs, allerdings in etwas geringerem Umfang als es bei den osteolytischen Metastasen der Fall ist. In der Gesamtpopulation der Brustkrebs-Patientinnen war die Anzahl der SREs in der Zoledronat- und PamidronatGruppe vergleichbar $(43 \%$ vs. $45 \%$; $\mathrm{p}=$ 0,309). In der Subgruppe der Patientinnen mit osteolytischen Läsionen traten jedoch bei den mit Zoledronat behandelten Frauen $17 \%$ weniger SREs auf als in der Pamidronat-Gruppe ( $48 \%$ vs. $58 \%$; $p=0,58)$. Bei Patientinnen, die mit Pamidronat behandelt wurden, trat die erste SRE bereits nach 174 Tagen auf, im Zoledronat-Arm hingegen erst nach 310 Tagen. Die komplikationsfreie Zeit unter Zoledronat konnte somit signifikant um mehr als 4,5 Monate verlängert werden (Abb. 1).

Die SMR war bei allen Patientinnen, die mit Zoledronat behandelten wurden, deutlich niedriger als bei den Patienten in der Pamidronat-Gruppe $(0,98$ vs. 1,55 Ereignisse/Patient/Jahr; $p=0,073)$. Statistische Signifikanz $(p=0,008)$ erreichte dieser Unterschied in der Subgruppe der Patientinnen mit osteolytischen Läsionen. Die SMR betrug hier in der Gruppe der mit $4 \mathrm{mg}$ Zoledronat behandelten Patientinnen 1,16 gegenüber 2,36 in der Pamidronat-Gruppe (Abb. 2).

\section{Mit Zoledronat geringeres Risiko für das Auftreten von Skelett- komplikationen}

Durch die «Multiple event»-Analyse wurde gezeigt, dass Zoledronat im Vergleich zu Pa- 


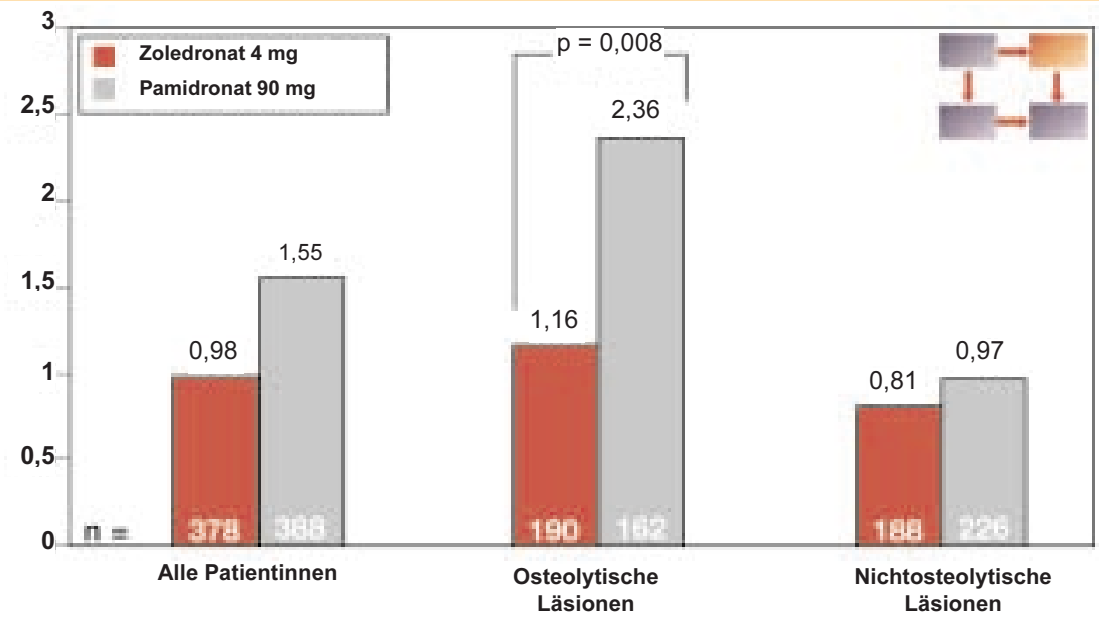

Abb. 2. SMR nach Therapiegruppe und Läsionstyp.

midronat das Risiko für SREs signifikant senkt. In der Gesamtpopulation der Patientinnen mit Mammakarzinom wurde es durch die Behandlung mit $4 \mathrm{mg}$ Zoledronat um $20 \%$ gegenüber einer Behandlung mit $90 \mathrm{mg}$ Pamidronat verringert. In der Subgruppe der Patientinnen mit osteolytischen Läsionen konnte sogar eine Senkung des Risikos um $30 \%$ erreicht werden (Hazard-Ratio $=0,704$, $\mathrm{p}=0,010)$ (Abb. 3)

Die Daten der beim ESMO vorgestellten Phase-III-Studie zeigen eindrucksvoll, dass bei Patientinnen mit Brustkrebs und mindestens einer osteolytischen Läsion $4 \mathrm{mg}$ Zoledronat in der Reduktion von SREs gegenüber $90 \mathrm{mg}$ Pamidronat signifikant effektiver sind. Dies ist besonders vor dem Hintergrund zu bewerten, dass osteolytische Läsio- nen ein höheren Risiko für SREs bergen als nichtosteolytische Läsionen. Für alle Patientinnen mit Mammakarzinom wird unter Zoledronat die Notwendigkeit einer palliativen Radiatio des Knochens signifikant gegenüber Pamidronat reduziert. Bei gleich guter Verträglichkeit kann Zoledronat als 15-minütige Infusion gegenüber der 2-stündigen Gabe von Pamidronat verabreicht werden. Zusammenfassend zeigten sich zudem in der osteolytischen Subgruppe folgende Ergebnisse: Weniger Patientinnen erlitten eine SRE, das Auftreten der ersten SRE konnte signifikant um mehr als 4,5 Monate verzögert werden und das Risiko, eine SRE zu erleiden, wurde durch die Therapie mit Zoledronat signifikant um 30\% im Vergleich zur Pamidronat-Therapie gesenkt.

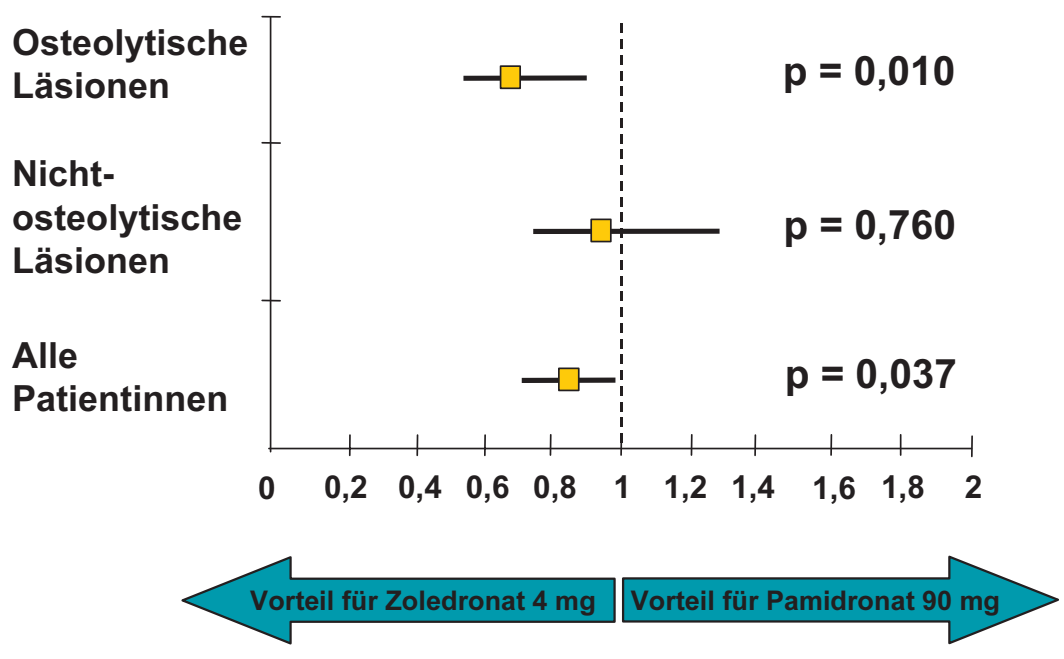

Abb. 3. Relatives Risiko für Mammakarzinom-Patientinnen, eine SRE zu erleiden (Hazard-Ratio, Konfidenzintervall 95\%).

\section{Weiterführende Literatur}

Jemal A, Thomas A, Murray T, Thun M: Cancer statistics, 2002. CA Cancer J Clin 2002:52:23-47.

Domichek SM, Younger J, Finkelstein DM, Seiden MV: Predictors of skeletal complications in patients with metastatic breast carcinoma. Cancer 2000;89:363-368.

Coleman RE: Skeletal complications of malignacy. Cancer 1997;80(suppl):1588-1594.

Hillner BE, Ingle JN, Berenson JR, et al: American Society of Clinical Oncology guideline on the role of bisphosphonates in breast cancer. American Society of Clinical Oncology Bisphosphonates Expert Panel. J Clin Oncol 2000;18: 1378 1391.

Hortobagyi GN, Theriault RL, Porter L, et al: Efficacy of pamidronate in reducing skeletal complications in patients with dronst in reducing skeletal con breast cancer and lytic bone metastases. Protocol 19 Aredia Breast Cancer Study Group. N Engl J Med 1996; 335 1785-1791.

Theriault RL, Lipton A, Hortobagyi GN, et al: Pamidronate re duces skeletal morbidity in women with advanced breast cancer and lytic bone lesions: A randomized, placebo-controlled trial. Protocol 18 Aredia Breast Cancer Study Group. J Clin Oncol 1999:17:846-854.

Rosen LS, Gordon D, Kaminski M, et al: Zoledronic acid versus pamidronate in the treatment of skeletal metastases in patients with breast cancer or osteolytic lesions of multiple myeloma. A phase III, double-blind, comparative trial. Cancer J 2001; 7: 377-387.

Garnero P: Markers of bone turnover in prostate cancer. Cancer Treat Rev 2001;27:187-192.

\section{Impressum}

Therapie von Knochenmetastasen beim Mammakarzinom - Zoledronat senkt Skelettkomplikationen osteolytischer Läsionen wirksamer als Pamidronat

Pharma Forum in Onkologie 25/6/02

Deutschland

S. Karger $\mathrm{GmbH}$

Lörracher Straße 16 a

D-79115 Freiburg

Tel. +49761452070

Fax +497614520714

E-mail Information@Karger.de

Übrige Länder

S.Karger AG

Allschwilerstrasse 10

$\mathrm{CH}-4009$ Basel

Tel. +4 613061111

Fax +4 613061234

E-mail Karger@Karger.ch

Mit freundlicher Unterstützung von Novartis Pharma GmbH, Nürnberg

Der Verlag und die Herausgeber der Zeitschrift übernehmen keine Verantwortung für diese Rubrik.

(c) 2002 by S. Karger Verlag

Verlag für Medizin und

Naturwissenschaften $\mathrm{GmbH}$

Lörracher Straße 16a, D-79115 Freiburg

Die Wiedergabe von Gebrauchsnamen, Handelsnamen, Warenbezeichnungen usw. in dieser Zeitschrift berechtigt auch ohne besondere Kennzeichnung nicht zur Annahme, dass solche Namen im Sinne der Warenzeichen- und Markenschutzim Sinne der Warenzeichen- und Markenschutz-
Gesetzgebung als frei zu betrachten wären und daher von jedermann benutzt werden dürfen. Für Angaben der Dosierungsanweisungen und Applikationsformen kann vom Verlag keine Gewähr nder im Einzellfall anhand anderer Literaturstellen auf ihre Richtigkeit überprüft werden. 